\title{
Aspectos cognitivos do falar em público: validação de uma escala de autoavaliação para universitários brasileiros
}

\author{
Cognitive aspects of public speaking: validation of a self-assessment scale for Brazilian university students
}

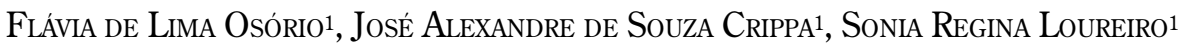 \\ 'Departamento de Neurociências e Comportamento - Faculdade de Medicina de Ribeirão Preto, Universidade de São Paulo (FMRP-USP); INCT Medicina Translacional.
}

Recebido: 20/7/2011 - Aceito: 01/12/2011

\begin{abstract}
Resumo
Contexto: O falar em público é o medo mais prevalente na população geral e no Transtorno de Ansiedade Social (TAS). Assim sendo, estudos que dimensionem essa situação específica são necessários. Objetivos: Validar a Self Statements during Public Speaking (SSPS) em amostra da população geral de estudantes universitários ( $\mathrm{PG}-\mathrm{n}=2.314)$, casos $(\mathrm{C}-\mathrm{n}=88)$ e não casos de TAS $(\mathrm{NC}-\mathrm{n}=90)$ do Brasil. Métodos: Conduziu-se o estudo em duas fases: a) preenchimento dos questionários autoaplicados em sala de aula; b) participação em entrevista telefônica e ao vivo. Resultados: Evidenciaram-se correlações baixas/moderadas entre SSPS e Inventário de Fobia Social ( $\mathrm{PG}=0,22-0,65 ; \mathrm{C}=0,28-0,32 ; \mathrm{NC}=0,21-0,30)$, Inventário de Ansiedade de Beck $(\mathrm{PG}=0,18-0,53 ; \mathrm{C}=0,25-0,33 ; \mathrm{NC}=$ 0,22-0,25) e Escala Breve de Fobia Social ( $C=$ não significativa, $\mathrm{NC}=0,23-0,31)$ nas diferentes amostras, especialmente para a PG. A análise fatorial apontou a presença de dois fatores, associados à autoavaliação positiva e negativa. O estudo da validade discriminativa evidenciou a capacidade da SSPS de discriminar os casos dos não casos de TAS. Conclusão: A SSPS é adequada para uso no contexto brasileiro, sendo que a subescala autoavaliação positiva parece ser mais efetiva para a avaliação de amostras identificadas ou suspeitas de TAS e a subescala autoavaliação negativa ter uma característica mais rastreadora quando aplicada em amostras da população geral.
\end{abstract}

Osório FL, et al. / Rev Psiq Clín. 2012;39(2):48-53

Palavras-chave: Falar em público, ansiedade social, validação, escala.

\begin{abstract}
Background: Fear of public speaking is the most prevalent fear in the general population and among persons with a social anxiety disorder (SAD). Nevertheless, studies about the assessment of this specific phenomenon are still needed. Objectives: To examine the validity of the Self Statements during Public Speaking (SSPS) scale in a general population of Brazilian university students (GP, $n=2314)$, in an SAD sample $(\mathrm{n}=88)$, and in a non-SAD sample $(\mathrm{n}=90)$. Methods: The study was conducted in two phases: a) completing a self-administered questionnaire in the classroom, b) participating in a telephone and face-to-face interview. Results: The SSPS showed low/median correlations with the Social Phobia Inventory $(\mathrm{GP}=0.22-0.65 ; \mathrm{SAD}=0.28-0.32 ;$ non-SAD $=0.21-0.30)$, the Beck Anxiety Inventory $(\mathrm{GP}=0.18-0.53$; SAD $=0.25-0.33$; non-SAD $=0.22-0.25)$, and the Brief Social Phobia Scale $(\mathrm{SAD}=$ not significant, non-SAD =0.23-0.31) in all the samples, especially in the GP sample. Factorial analysis indicated the presence of two factors associated with positive and negative self-assessment. The study of discriminative validity demonstrated that the SSPS has the ability to differentiate between cases and non-cases of SAD. Discussion: The SSPS is an adequate instrument to be used within the Brazilian context. The positive self-assessment subscale seems to be more effective for the evaluation of SAD samples. The negative self-assessment subscale appears to have a better screening characteristic in the general population of university students.
\end{abstract}

Osório FL, et al. / Rev Psiq Clín. 2012;39(2):48-53

Keywords: Public speaking, social anxiety, validation, scale.

\section{Introdução}

Estudos epidemiológicos evidenciaram que o medo de falar em público é o mais prevalente na população gerall,2, e sua prevalência independe de gênero, etnia e idade ${ }^{3}$, o que tem despertado e estimulado estudos que avaliem e dimensionem tal situação específica.

No transtorno de ansiedade social (TAS), que se caracteriza pelo medo e evitação de situações sociais e de performance, o falar em público também tem sido apontado como o medo mais prevalente nos portadores de TAS generalizado e, principalmente, nos portadores de TAS circunscrito ou não generalizado ${ }^{4-6}$, salientando a importância do componente cognitivo na etiologia desse transtorno.

Em contrapartida, há uma carência de instrumentos padronizados e validados para a avaliação dos aspectos cognitivos do TAS ${ }^{7,8}$, destacando-se a Self Statements during Public Speaking Scale (SSPS) ${ }^{9}$. A SSPS tem como fundamento as teorias cognitivas, que pressupõem que a ansiedade social é resultado de uma percepção negativa de si e dos outros em relação a si. Trata-se de escala autoa- plicável, composta por duas subescalas: a de autoavaliação positiva e a de autoavaliação negativa, cada qual com cinco itens pontuados numa escala de 0 a 5 .

As qualidades psicométricas da SSPS foram inicialmente avaliadas por Hofmann e DiBartolo9 ${ }^{9}$ em uma amostra da população geral de universitárias e não universitárias saudáveis e em casos de TAS, constatando a adequação do instrumento. Posteriormente, a SSPS fora traduzida e adaptada para o alemão $0^{10}$, para o espanhol ${ }^{11-12}$ e para o português do Brasili13, e os estudos psicométricos iniciais dessas versões, com amostras mais amplas e diversificadas, mostraram-se promissores.

Especificamente quanto à versão para o português, analisou-se inicialmente a consistência interna e dos itens, em uma população de universitários brasileiros, de ambos os sexos, a qual se mostrou adequada $^{13}$. Objetivando-se dar continuidade a essa análise, o presente estudo propõe-se a avaliar diferentes indicadores de validade da SSPS em amostra da população geral de universitários e em amostra clínica e não clínica de TAS. 


\section{Métodos}

O estudo foi conduzido em duas fases distintas. O objetivo da fase 1 foi avaliar as propriedades psicométricas da SSPS em uma amostra da população geral e o da fase 2 , em uma amostra de casos e não casos de TAS.

\section{Sujeitos}

Fase 1 - 2.614 estudantes universitários de duas universidades brasileiras, uma pública e uma privada, foram convidados a responder, em sala de aula, a instrumentos de autoavaliação. Os critérios de inclusão foram: ambos os sexos e idade entre 17 e 35 anos, enquanto os critérios de exclusão utilizados foram: uso de neurolépticos e resposta incorreta e/ou incompleta aos instrumentos. Tendo como parâmetro esses critérios, a amostra final foi composta por 2.314 sujeitos.

Fase 2 - Com base na MiniSPIN (MS) ${ }^{14}$, uma versão reduzida do Inventário de Fobia Social (SPIN), com altos indicadores de sensibilidade para rastreamento dos casos de TAS, e no módulo F da Entrevista Estruturada para o DSM-IV (SCID-IV) ${ }^{15}$, os sujeitos da fase 1 foram selecionados por conveniência para compor a amostra dessa outra fase do estudo $(\mathrm{N}=372)$. Desses, 95 não aceitaram continuar a participar do estudo e 73 não foram localizados, sendo a amostra final composta por 204 universitários. Os critérios de inclusão no grupo casos de TAS foi: a) escore na MS maior ou igual a 6, conforme proposto no estudo original de Connor et al.14; b) diagnóstico de TAS baseado na SCID-IV; c) ausência de comorbidades psiquiátricas avaliadas por meio da SCID-IV; d) preenchimento correto dos instrumentos. Os critérios de inclusão para o grupo não casos de TAS foram: a) escore na MS de 0 ou 1, em acordo com os critérios de Connor et al. ${ }^{14}$; b) ausência de qualquer diagnóstico psiquiátrico avaliado por meio da SCID-IV; c) preenchimento correto dos instrumentos. Com base nesses critérios, uma amostra de 178 sujeitos foi obtida, sendo 88 sujeitos do grupo casos de TAS e 90 do grupo não casos de TAS.

\section{Materiais}

Foram utilizados os seguintes instrumentos para as diferentes fases da coleta de dados:

a) Self Statements Public Speaking (SSPS) ${ }^{9}$ - versão traduzida e adaptada para o Brasil por Osório et al. ${ }^{13}$. Instrumento autoaplicado para avaliação do falar em público. É composto por 10 itens pontuados em uma escala likert de 0 (discordo totalmente) a 5 (concordo inteiramente), que tem por objetivo avaliar a autopercepção do desempenho na situação específica de falar em público. É dividida em duas subescalas: autoavaliação positiva (itens $1,3,5,6,9)$ e autoavaliação negativa (itens $2,4,7,8,10$ );

b) Social Phobia Inventory (SPIN) ${ }^{16}$ - versão traduzida e adaptada para o português do Brasil por Osório et al. ${ }^{17}$. Instrumento autoaplicado para avaliação do TAS, composto por 17 itens pontuados em uma escala likert de 5 pontos;

c) Mini Social Phobia Inventory (MS) ${ }^{14}$ - versão traduzida e adaptada para o português do Brasil por Osório et al. ${ }^{18}$. Instrumento de rastreamento do TAS, composto por três itens, pontuados em uma escala likert de 0 a 4 ;

d) Beck Anxiety Inventory (BAI) ${ }^{19}$ - versão traduzida e adaptada para o português do Brasil por Cunha ${ }^{20}$ - instrumento autoaplicado para avaliação dos aspectos gerais de ansiedade, composto por 21 itens pontuados em uma escala likert de 5 pontos;

e) Brief Social Phobia Scale (BSPS) 21 - versão traduzida e adaptada para o português do Brasil por Osório et al. ${ }^{22}$ - instrumento de heteroavaliação do TAS, composto por 18 itens, pontuados em uma escala likert de 5 pontos;

f) Questionário de Identificação - instrumento construído para o estudo em questão objetivando coletar dados relativos à caracterização sociodemográfica e clínica da amostra;

g) Entrevista Clínica Estruturada para o DSM-IV (SCID-IV) ${ }^{15}$ - versão traduzida e adaptada para o português por Del-Ben et al. ${ }^{23}$
- instrumento utilizado para a elaboração de diagnósticos clínicos psiquiátricos baseados no DSM-IV, composto por módulos, em um total de 10, que podem ser aplicados de forma independente ou combinada.

\section{Procedimentos}

O estudo foi aprovado pelo Comitê de Ética em Pesquisa do Hospital das Clínicas da Faculdade de Medicina de Ribeirão Preto da Universidade de São Paulo ( $\left.{ }^{\circ} 11570-2003\right)$.

Fase 1 - Contataram-se estudantes universitários de duas universidades do Brasil, sendo uma pública e outra privada. Em sala de aula, os sujeitos que concordaram em participar do estudo responderam aos instrumentos de autoavaliação: SSPS, SPIN/MS e BAI.

Fase 2 - Selecionaram-se inicialmente sujeitos que preencheram critérios para MS positiva e outros para MS negativa. Contataram-se eles por telefone, visando confirmar a continuidade da participação no estudo, e àqueles que concordaram em participar aplicou-se, por meio de entrevista telefônica, o módulo F da SCID-IV, relativa ao TAS. A validade de tal metodologia aplicada por telefone e ao vivo foi anteriormente testada por Crippa et al. ${ }^{24} \mathrm{e}$ mostrou-se bastante eficaz $(k a p p a=0,80)$.

Posteriormente, os sujeitos que preencheram os critérios relativos à pontuação na MS e módulo F da SCID-IV para o grupo de casos e não casos de FS foram convidados a participar de uma entrevista ao vivo para a aplicação da SCID-IV completa, objetivando a avaliação da presença de outros transtornos psiquiátricos (comorbidades) e a aplicação da BSPS, instrumento de heteroavaliação do TAS. Essa entrevista foi conduzida por avaliados experientes e previamente treinados. Nessa ocasião, os sujeitos preencheram novamente, de forma individual, os instrumentos autoaplicados: SSPS, SPIN/MS e BAI.

\section{Codificação e tratamento dos dados}

Os dados foram alocados em um banco de dados e submetidos à análise utilizando-se o pacote estatístico SPSS, versão 13.0 (SPSS Incorporation, 2001).

A análise dos dados demográficos e clínicos da amostra estudada foi realizada por meio da aplicação de testes estatísticos descritivos e paramétricos. Para a comparação de grupos, foi utilizado o teste $t$ de Student e o teste do qui-quadrado.

As seguintes técnicas foram utilizadas para o estudo de validade da SSPS:

- coeficiente de correlação de Pearson para os escores totais e subescalas da SSPS, BAI, SPIN e BSPS, visando à avaliação da validade concorrente entre os instrumentos. A magnitude das correlações encontradas foram classificadas em: 0 a 0,25: fraca; 0,26 a 0,50: moderada; 0,51 a 0,70: forte, acima de 0,71: muito forte 25 ;

- teste $t$ de Student para análise da validade discriminativa em função das variáveis: sexo e grupo'26;

- Análise Fatorial Exploratória por meio da técnica de análise dos componentes principais com rotação varimax, visando à avaliação da validade de construto da escala. Os critérios utilizados para a composição dos fatores foram: índice de Kaiser-Meyer-Olkin (KMO) acima de 0,60; teste de esfericidade de Bartlett significativo, autovalores acima de 1; percentual de variância explicada pelos fatores de aproximadamente $60 \%$; e carga fatorial mínima de aproximadamente $0,40^{27}$.

Adotou-se em todas as análises nível de significância de $\mathrm{p} \leq 0,05$.

\section{Resultados}

\section{Caracterização sociodemográfica da amostra}

A amostra final da fase 1 do estudo foi composta por 2.314 estudantes, sendo $55,3 \%$ da universidade privada e $44,7 \%$ da pública. Observou-se um pequeno predomínio do sexo feminino $(55,8 \%)$, e a média de idade da amostra ficou em torno dos 21,4 anos (DP = $3,3)$. A maior parte dos sujeitos das duas instituições não realizava 
outras atividades de trabalho além do estudo (78,9\%). Predominaram alunos dos cursos da área de Biológicas $(60 \%)$, e a maior parte deles $(61,5 \%)$ frequentava os anos iniciais $\left(1^{\circ}\right.$ e $\left.2^{\circ}\right)$ dos cursos.

Compuseram a amostra final da fase 2 do estudo 178 sujeitos, sendo 88 casos de TAS e 90 não casos de TAS. Nessa amostra também predominaram sujeitos do sexo feminino $(61,8 \%)$, e a idade média dos sujeitos ficou em torno de 21,2 anos. Aproximadamente, $90 \%$ dos sujeitos apenas estudavam, e do total, 75,6\% dos alunos eram da universidade pública e $24,4 \%$ da privada. Predominaram também alunos dos cursos da área de Biológicas (54,3\%), cursando os primeiros e segundos anos dos diferentes cursos (68\%). Analisando-se a amostra em função da presença ou ausência de TAS, não se encontraram diferenças significativas quanto às variáveis sociodemográficas, evidenciando a homogeneidade dos dois subgrupos: casos e não casos de TAS (Tabela 1).

\section{Validade concorrente e divergente}

Dada a ausência de instrumentos específicos para a avaliação do falar em público validados para o contexto estudado, analisou-se a validade concorrente da SSPS com um instrumento de avaliação geral da ansiedade e com outros dois instrumentos específicos para avaliação de diferentes aspectos da ansiedade social.

Quanto às correlações com o BAI, o instrumento geral de ansiedade, observou-se que, na amostra da população geral, todos os itens da SSPS guardaram correlações significativas com os itens $(0,02$ a 0,$35 ; \mathrm{p}<0,01)$ e escore total $(0,07$ a 0,$43 ; \mathrm{p}<0,01)$ da BAI, com magnitude considerada fraca a moderada, sendo o item 10 da SSPS ("Eu me sinto desajeitado e tolo; certamente eles vão notar") aquele que apresentou as maiores correlações. Para as amostras clínicas de casos e não casos de TAS, o número de correlações foi menor e de magnitude inferior, apontando para a especificidade da SSPS em relação ao BAI, o que de certa forma evidencia a sua validade divergente.

As correlações da SSPS e de suas subescalas com o escore total e as subescalas dos instrumentos tomados como referência para estudo da validade concorrente estão apresentadas na tabela 2 .

Observa-se que, para a amostra da população geral, a subescala de autoavaliação negativa foi aquela que guardou as maiores correlações $(0,37-0,53 ; p<0,01)$ com o BAI, correlações essas classificadas como moderada/fortes. A subescala de autoavaliação positiva mostrou correlações significativas, porém com níveis considerados fracos $(-0,18$ a - 0,$24 ; \mathrm{p}<0,01)$. Tomando-se como referência o BAI, a escala total

Tabela 1. Caracterização sociodemográfica da amostra final da fase 2, em função da ausência ou presença de TAS ( $N=178)$

\begin{tabular}{|c|c|c|c|c|c|}
\hline \multicolumn{2}{|l|}{ Variáveis } & $\begin{array}{l}\text { Casos } \\
(N=88)\end{array}$ & $\begin{array}{c}\text { Não casos } \\
(\mathrm{N}=90)\end{array}$ & $\begin{array}{c}\text { Total } \\
(N=178)\end{array}$ & Estatística \\
\hline \multirow[t]{2}{*}{ Sexo } & Feminino N (\%) & $60(68,2)$ & $50(55,6)$ & $110(61,8)$ & \multirow{2}{*}{$\begin{aligned} \chi^{2} & =3.005 \\
p & =0,83\end{aligned}$} \\
\hline & Masculino N (\%) & $28(31,8)$ & $40(44,4)$ & $68(38,2)$ & \\
\hline Idade & \begin{tabular}{|l|} 
Média \\
(DP) \\
\end{tabular} & $\begin{array}{l}21,5 \\
(2,9) \\
\end{array}$ & $\begin{array}{l}20,9 \\
(2,5) \\
\end{array}$ & $\begin{array}{l}21,2 \\
(2,7) \\
\end{array}$ & $\begin{array}{l}t=1.438 \\
p=0,15\end{array}$ \\
\hline \multirow[t]{2}{*}{ Atividade profissional } & Estuda $\mathrm{N}(\%)$ & $81(92,0)$ & $79(87,8)$ & $160(89,9)$ & \multirow{2}{*}{$\begin{aligned} \chi^{2} & =0,892 \\
p & =0,35\end{aligned}$} \\
\hline & Estuda + trabalha N (\%) & $07(8,0)$ & $11(12,2)$ & $18(10,1)$ & \\
\hline \multirow[t]{2}{*}{ Universidade } & Pública N (\%) & $63(72,1)$ & $72(78,9)$ & $133(75,6)$ & \multirow{2}{*}{$\begin{aligned} \chi^{2} & =1.100 \\
p & =0,29\end{aligned}$} \\
\hline & Privada N (\%) & $24(27,9)$ & $19(21,1)$ & $43(24,4)$ & \\
\hline \multirow{3}{*}{$\begin{array}{l}\text { Área do } \\
\text { curso }\end{array}$} & Exatas $(\%)$ & $34(31,4)$ & $28(31,4)$ & $62(34,3)$ & \multirow{3}{*}{$\begin{aligned} \chi^{2} & =0,643 \\
p & =0,73\end{aligned}$} \\
\hline & Humanas N (\%) & $10(11,2)$ & $10(11,6)$ & $20(11,4)$ & \\
\hline & Biológicas N (\%) & $46(51,7)$ & $50(57,0)$ & $96(54,3)$ & \\
\hline \multirow{4}{*}{ Ano do curso } & $1^{\circ} \mathrm{N}(\%)$ & $28(31,4)$ & $38(41,6)$ & $66(36,6)$ & \multirow{4}{*}{$\begin{aligned} \chi^{2} & =3.221 \\
p & =0,36\end{aligned}$} \\
\hline & $2^{\circ} \mathrm{N}(\%)$ & $29(32,6)$ & $27(30,3)$ & $56(31,4)$ & \\
\hline & $3^{\circ} \mathrm{N}(\%)$ & $21(24,4)$ & $20(22,5)$ & $41(23,4)$ & \\
\hline & $4^{\circ} \mathrm{N}(\%)$ & $10(11,6)$ & $5(5,6)$ & $15(8,6)$ & \\
\hline
\end{tabular}

N: frequência; \%: porcentagem.

Tabela 2. Valores relativos ao estudo da validade concorrente/divergente da Self Statements during Public Speaking Scale (SSPS) e suas subescalas com o Beck Anxiety Inventory (BAI), Social Phobia Inventory (SPIN) e Brief Social Phobia Scale (BSPS) nas diferentes amostras estudadas

\begin{tabular}{|c|c|c|c|c|c|c|c|}
\hline & \multicolumn{6}{|c|}{ SSPS } \\
\hline & & \multicolumn{2}{|c|}{$\begin{array}{l}\text { População geral } \\
\qquad(\mathrm{N}=2.314)\end{array}$} & \multicolumn{2}{|c|}{$\begin{array}{c}\text { Casos TAS } \\
(\mathrm{N}=88)\end{array}$} & \multicolumn{2}{|c|}{$\begin{array}{l}\text { Não casos TAS } \\
(\mathrm{N}=90)\end{array}$} \\
\hline & & $\mathrm{SP}$ & SN & SP & SN & $S P$ & SN \\
\hline \multirow[t]{5}{*}{ BAI } & ET & $-0.24^{* *}$ & $0.52^{* *}$ & -0.14 & $0.29^{* *}$ & $-0.22^{*}$ & 0.16 \\
\hline & Neurofisiológica & $-0.21^{* *}$ & $0.43^{* *}$ & -0.09 & $0.28^{* *}$ & -0.19 & 0.16 \\
\hline & Subjetiva & $-0.23^{* *}$ & $0.53^{* *}$ & -0.07 & $0.29^{* *}$ & $-0.25^{*}$ & 0.16 \\
\hline & Pânico & $-0.19^{* *}$ & $0.37^{* *}$ & -0.04 & 0.20 & -0.10 & 0.07 \\
\hline & Autonômica & $-0.18^{* *}$ & $0.39 * *$ & $-0.33^{* *}$ & 0.15 & -0.11 & 0.07 \\
\hline \multirow[t]{4}{*}{ SPIN } & ET & $-0.31^{* *}$ & $0.65^{* *}$ & $-0.31^{* *}$ & 0.12 & $-0.28^{* *}$ & $0.21^{*}$ \\
\hline & Medo & $-0.30^{* *}$ & $0.62^{* *}$ & $-0.30^{* *}$ & 0.13 & $-0.25^{* *}$ & $0.21^{*}$ \\
\hline & Evitação & $-0.32^{* *}$ & $0.60^{* *}$ & $-0.32^{* *}$ & 0.13 & $-0.30^{* *}$ & 0.19 \\
\hline & Sintomas fisiológicos & $-0.22^{* *}$ & $0.52^{* *}$ & -0.21 & 0.05 & -0.30 & -0.19 \\
\hline \multirow[t]{4}{*}{ BSPS } & ET & --- & --- & -0.19 & 0.14 & $-0.31^{* *}$ & 0.15 \\
\hline & Medo & --- & --- & -0.14 & 0.14 & $-0.30^{* *}$ & 0.11 \\
\hline & Evitação & --- & --- & -0.19 & 0.15 & $-0.27^{* *}$ & 0.16 \\
\hline & Sintomas fisiológicos & --- & --- & -0.13 & 0.03 & -0.19 & 0.11 \\
\hline
\end{tabular}

${ }^{*}: p<0,05 ;{ }^{* *}: p<0,01 ;$ SP: subescala positiva; SN: subescala negativa; TAS: transtorno de ansiedade social. 
e a subescala subjetiva foram aquelas que guardaram as melhores correlações com a SSPS.

Para a amostra clínica de casos de TAS, as correlações mais significativas, porém classificadas como fracas, entre a SSPS e o BAI foram identificadas para a subescala de autoavaliação negativa, e para a subescala de pânico do BAI, nenhuma correlação significativa foi evidenciada. Para a amostra de não casos, as correlações mais significativas foram entre a subescala de autoavaliação positiva da SSPS e a escala total e a subescala subjetiva do BAI, mesmo assim com magnitude fraca.

No que diz respeito às correlações entre a SSPS e a SPIN, observou-se que, na amostra da população geral, todos os itens desses instrumentos correlacionaram-se entre si e com o escore total, com valores variando entre 0,03 e $0,57(\mathrm{p}<0,01)$, sendo novamente o item 10 da SSPS aquele com índices de melhores correlações. Para a amostra de casos e não casos, o número de correlações entre os itens foi menor e menos expressivo, e nos casos de TAS, o item 3 da subescala positiva ("Esta é uma situação difícil, mas posso dar conta dela") apresentou as maiores e melhores correlações, e na amostra de não casos o item 5 , também da subescala positiva ("Mesmo que não dê certo, não é o fim do mundo").

Quanto às subescalas, os dados da tabela 2 mostram que para a população geral a melhor correlação evidenciada foi em relação à subescala de autoavaliação negativa da SSPS e o SPIN total $(\mathrm{r}=$ $0,65 ; p<0,01)$, correlação essa classificada como forte. No geral, foi a subescala de autoavaliação negativa que também mostrou as melhores correlações com as subescalas do SPIN ( $\mathrm{r}=0,52$ a 0,$62 ; \mathrm{p}$ $<0,01)$. Já as correlações da subescala de autoavaliação positiva da SSPS com o SPIN foram inferiores, consideradas fracas/moderadas, com valores entre $-0,22$ e $-0,32(\mathrm{p}<0,01)$. Quanto ao SPIN, tanto a escala total como as subescalas de medo e evitação apresentaram as correlações mais significativas com a SSPS.

As maiores correlações observadas na amostra de casos foram entre a subescala positiva da SSPS e a escala total e subescalas de medo e evitação do SPIN, correlações essas classificadas como moderadas. Na amostra de não casos, as maiores correlações foram entre a subescala positiva da SSPS e escala total e subescalas do SPIN, mantendo-se classificadas qualitativamente como fracas, tendendo a moderadas. A subescala negativa da SSPS foi aquela que menos guardou correlações com o SPIN nessas amostras específicas. Com relação ao SPIN, a subescala de sintomas fisiológicos foi aquela com as menores correlações.

Quanto ao estudo das correlações entre a SSPS e a BSPS, realizado apenas com as amostras de casos e não casos, identificaram-se poucas correlações significativas entre os itens e o escore total, apontando para a validade divergente dos instrumentos e a especificidade da SSPS em relação à BSPS, uma escala para heteroavaliação dos aspectos gerais do TAS. Esses dados são confirmados quando se observa na tabela 2 a correlação entre as subescalas, já que não se evidenciou nenhuma correlação significativa para a amostra de casos entre as duas escalas. Para a amostra de não casos, as correlações mais significativas da BSPS foram com a subescala de autoavaliação positiva da SSPS, correlações essas de ordem negativa, classificadas no geral como fracas, tendendo a moderadas $(-0,23 \mathrm{a}-0,31 ; \mathrm{p}<0,01)$.

\section{Validade de construto - Análise fatorial}

A análise fatorial da SSPS foi realizada separadamente para as amostras da população geral e de casos de TAS. Para a amostra da população geral, extraíram-se, pela técnica dos componentes principais com rotação varimax, dois fatores, que responderam conjuntamente por $52 \%$ da variância dos dados. O fator 1 , denominado de autoavaliação negativa, ficou composto por cinco itens (itens: $2,4,7,8$ e 10), apresentando consistência interna de 0,78 . O fator 2 também foi composto por cinco itens (itens: $1,3,5,6$ e 9), sendo denominado autoavaliação positiva, com consistência interna de 0,80 .

Da mesma forma, para a amostra de casos, os dois fatores foram extraídos, sendo cada um composto pelos mesmos itens dos fatores da amostra da população geral, e assim recebendo a mesma denomi- nação. Para essa amostra, os dois fatores, conjuntamente, explicaram $62,7 \%$ da variância dos dados, e cada qual apresentou consistência interna adequada (fator 1: $\alpha=0,76$; fator 2 : $\alpha=0,83$ ).

\section{Validade discriminativa}

Analisou-se a capacidade da SSPS em discriminar os sujeitos em função das variáveis sexo e grupo (caso e não caso de TAS).

Quanto ao sexo, na população geral, apenas a subescala negativa mostrou-se discriminativa, por meio do teste $t$ de Student $(\mathrm{t}=-4,802$; $\mathrm{p}<0,001)$. Nessa subescala, os sujeitos do sexo feminino mostraram menor média de pontuação, ou seja, maior avaliação negativa de si (pontuação invertida). Para as amostras específicas, não se encontraram diferenças significativas entre os sexos.

Quanto à capacidade de discriminar casos e não casos de TAS, os sujeitos com TAS, por meio da SSPS, denotaram médias de pontuação bastante inferiores aos não casos, evidenciando uma autoavaliação menos positiva e mais negativa de si (subescala positiva: $t=6,469$; $\mathrm{p}<0,001$; subescala negativa: $\mathrm{t}=4,234 ; \mathrm{p}<0,001)$.

\section{Discussão}

Os dados, no geral, mostraram o bom nível de adequação da SSPS. Em relação à validade concorrente, como já explicitado, esta foi estudada somente em relação a um instrumento geral de ansiedade (BAI) e dois instrumentos específicos para avaliação sintomática do TAS (SPIN e BSPS), haja vista que, até o momento da realização deste estudo, não existiam disponíveis para o nosso contexto escalas específicas para a avaliação do falar em público. Assim, teoricamente, o que se esperava em relação a essas análises é que fossem identificadas correlações medianas, uma vez que o medo de falar em público é um estressor psicossocial que desperta vivências de ansiedade, bem como está diretamente associado à sintomatologia do TAS.

Quanto às análises relativas à validade concorrente/divergente com o BAI, instrumento geral de avaliação da ansiedade, observou-se, na amostra da população geral, um maior número de correlações entre os itens da SSPS e o BAI, em comparação com a subamostra de casos e não casos, e tais correlações não foram específicas. Quanto às correlações entre as escalas totais e subescalas, as mesmas variaram na amostra da população geral de $-0,18$ a 0,53 , sendo as maiores entre a subescala negativa da SSPS e a subescala subjetiva do BAI.

Para os casos, as correlações mais significativas foram com a subescala negativa da SSPS, e para os não casos, com a subescala positiva. Tais valores vêm ao encontro da expectativa inicial de que existissem correlações entre esses dois instrumentos, mas que elas não fossem tão expressivas, pois a ansiedade, em suas diversas formas de manifestações, não se restringem ao falar em público.

Quanto às correlações com o SPIN, o que se identificou para a amostra da população geral é que todos os itens da SSPS guardaram relações significativas com o esse instrumento, sendo a mais significativa entre os itens que avaliam os aspectos relativos ao sentir-se bobo ou envergonhado e assim ser percebido pelo outro. Na população geral, os itens da subescala de autoavaliação positiva foram aqueles que menos se correlacionaram com o SPIN, relação essa de ordem negativa, denotando que tal avaliação favorável do desempenho e da capacidade pessoal guarda menores correlações com os sintomas do TAS, o que já não acontece com as avaliações de caráter negativo. Já para a amostra de casos e não casos de TAS, isso não ocorreu, e nessas amostras as correlações entre os itens não foram específicas, possivelmente sugerindo peculiaridades da amostra clínica. Ainda nessa direção, analisando-se as correlações entre as escalas totais e subescalas, a maior correlação encontrada na população geral foi entre a subescala negativa da SSPS e a escala total e subescalas do SPIN, e as menores entre a subescala positiva da SSPS e o SPIN, reforçando a afirmação acima de que os sintomas do TAS estão associados a um aumento da avaliação negativa de si, e que a subescala negativa guarda mais correlações com o SPIN, do que a subescala positiva, em acordo com o aporte teórico de construção do instrumento, o qual 
pressupõe que a ansiedade social é fruto de uma percepção negativa de si e dos outros em relação a si9.

Analisando as correlações entre as subescalas da SSPS e SPIN para as amostras de casos de TAS, observa-se que, diferente do anteriormente descrito, as correlações mais significativas foram encontradas entre a subescala positiva da SSPS e o SPIN, o mesmo ocorrendo para os não casos, expressando que, nessas amostras, os sintomas ligados ao TAS estão mais associados a uma diminuição da autoavaliação positiva do que a um aumento da avaliação negativa, pois em relação à subescala negativa da SSPS e o SPIN não se evidenciaram correlações específicas. Esses dados vão ao encontro do estudo de Rivero et al. ${ }^{12}$, com amostra de adolescentes espanhóis, em que foram encontrados indicadores mais consistentes de validade para a subescala positiva na amostra clínica. Pode-se pensar que, para a amostra de pessoas diagnosticadas como portadores do TAS, a diminuição da autoavaliação positiva reflete os prejuízos decorrentes do transtorno, sobrepondo-se à avaliação negativa. Assim, quanto ao estudo da validade concorrente com o SPIN, instrumento específico para a avaliação do TAS, encontraram-se correlações diversas nas diferentes amostras estudadas. $\mathrm{Na}$ população geral, os sintomas do TAS mostraram-se mais associados ao aumento da avaliação negativa de si e na amostra de casos e não casos, à diminuição da avaliação positiva. Esses resultados também são consistentes com um estudo recente no qual se observou, por meio de um paradigma de reconhecimento de expressão facial, que os casos de TAS eram hipersensíveis a estímulos de ameaça e de aprovação social 28 .

Em relação à validade concorrente com a BSPS, observou-se que para os casos não ocorreram correlações significativas e para os não casos as correlações foram não significativas ou pouco expressivas $(0,23$ a 0,31$)$. Em nenhuma das amostras, observaram-se correlações para a subescala negativa, denotando que essa subescala tem qualidades psicométricas inferiores na comparação com a subescala positiva para as amostras específicas de casos e não casos. Também se observaram pouquíssimas correlações entre os itens desses dois instrumentos. Considerando-se que o falar em público e os aspectos cognitivos associados a ele são apenas uma das facetas do TAS, as fracas correlações evidenciadas entre a BSPS e SSPS podem ser explicadas pela especificidade dos dois instrumentos e também pelas diferentes vertentes de avaliação (auto e hetero) utilizadas para o preenchimento deles, diminuindo, assim, a correlação entre eles.

No estudo original ${ }^{9}$, as correlações entre a SSPS e as escalas específicas para avaliação do TAS, a saber Fear Negative Evaluation (FNE), Schedule for Affective Disorders and Schizophrenia (SADS) e Social Phobia Anxiety Inventory (SPAI), evidenciaram valores próximos ao do estudo em questão com a amostra da população geral. $\mathrm{O}$ mesmo se pode dizer em relação ao estudo de Gerlach et al. (2007), que avaliou as correlações entre a SSPS e SPAI, Social Phobia Scale (SPS) e State-Trait Anxiety Inventory (STAI-T). Tais dados evidenciam que o medo de falar em público é um domínio relevante do TAS, mas que a ansiedade social não se manifesta exclusivamente nesse domínio. Assim como no presente estudo, no estudo de Gerlach et al..$^{10}$, com amostra de estudantes universitários, encontrou-se também uma maior associação entre a subescala negativa da SSPS e as diferentes medidas de avaliação do TAS, reforçando a hipótese de que na população geral os sintomas do TAS estariam associados a um aumento da avaliação negativa de si.

Também no estudo de Hofmann e DiBartolo9 ${ }^{9}$ a análise da validade divergente da SSPS, por meio da correlação com a BDI, apresentou valores de 0 a 0,21 , ou seja, pouco expressivos. No estudo em questão, tal validade pode ser evidenciada quando observada a correlação entre a SSPS e a subescala de pânico do BAI, que nas três amostras foi aquela que menos guardou correlações com a SSPS e suas subescalas. Também pode ainda ser evidenciada, por meio da própria correlação com os instrumentos BAI e SPIN, que ficou entre os níveis fraco e moderado, instrumentos esses com outras especificidades diferentes da SSPS.

Quanto à análise fatorial, assim como no estudo de Hofmann e DiBartolo', encontraram-se dois fatores. O primeiro fator é relativo à autoavaliação negativa com maior carga fatorial e maior porcentagem da variância, assim como no estudo original, em que os valores foram inclusive muito próximos. O segundo fator foi relativo à autoavaliação positiva com menor carga e menor porcentagem de variância, igualmente em concordância com o estudo original. Assim, comparativamente, nas duas amostras do presente estudo e no estudo original, não se observaram diferenças quanto à análise fatorial, reforçando a validade de construto do instrumento, como foi reforçado pelos estudos de Gerlach et al. ${ }^{10}$ e Gallego et al. ${ }^{11}$, em que a estrutura fatorial da SSPS foi replicada.

Em relação à validade discriminativa, observou-se que a SSPS também foi capaz de discriminar os grupos casos e não casos de TAS, apresentando os casos médias inferiores na pontuação final do instrumento, ou seja, uma menor avaliação positiva de si, tal qual já evidenciado por Rivero et al. ${ }^{12}$. Esse dado reforça os achados prévios da literatura ${ }^{2,5,6}$ de que o medo de falar em público é um domínio bastante relevante do TAS, sendo capaz de por si só, com alto grau de significância, diferenciar os sujeitos classificados como casos e não casos de TAS. Por outro lado, a capacidade da SSPS em identificar as diferenças entre os sexos, diferenças essas descritas na literatura prévia, em que as mulheres apresentam maior nível de sintomatologia em relação aos homens ${ }^{29}$, foi limitada e específica à subescala negativa quando da avaliação da população geral de universitários.

\section{Conclusão}

No geral, o estudo psicométrico da SSPS, realizado por meio da avaliação da validade concorrente, divergente, discriminativa, de construto e análise fatorial, demonstrou a adequação do instrumento para o uso na população brasileira, com qualidades psicométricas semelhantes às do estudo original. A subescala positiva parece ser mais efetiva para a avaliação de amostras identificadas ou suspeitas de TAS, diferentemente do estudo original da escala (Hofmann e DiBartolo, 2000) ${ }^{9}$, e a subescala negativa pareceu ter mais uma característica rastreadora quando aplicada em amostras da população geral.

A SSPS, na sua versão para o português do Brasil, poderá preencher uma necessidade no que diz respeito à avaliação sistemática do medo de falar em público, em estudos clínicos, e sobretudo em estudos experimentais, valorizando os aspectos cognitivos relacionados ao referido medo e, consequentemente, ao TAS, aspectos esses essenciais na etiologia do transtorno e não abarcados pelos demais instrumentos disponíveis na literatura para uso na população brasileira. Entretanto, estudos futuros avaliando as propriedades psicométricas da SSPS em diferentes populações e contextos são desejáveis e necessários.

\section{Referências}

1. Geer JH. The development of a scale to measure fear. Behav Res Ther. 1965;3:416-24

2. Stein MB, Walker JR, Forde DR. Public speaking fears in a community sample. Prevalence, impact on functioning and diagnostic classification. Arch Gen Psychiatry. 1996;53:169-74.

3. Phillips GC, Jones GE, Rieger ER, Snell JB. Normative data for the personal report of confidence as a speaker. J Anxiety Disord. 1997;11:215-20.

4. Stein MB, Walker JR, Forde DR. Setting diagnostic thresholds for social phobia: considerations from a community survey of social anxiety. Am J Psychiatry. 1994;151:408-12.

5. Furmark T, Tillfors M, Everez P, Marteinsdolter I, Gefvert O, Fredrikson M. Social phobia in the general population: prevalence and sociodemographic profile. Soc Psychiatry Psychiatr Epidemiol. 1999;34:416-24.

6. Baptista CA. Estudo da prevalência do transtorno de ansiedade social em estudantes universitários [dissertação]. Faculdade de Medicina de Ribeirão Preto da Universidade de São Paulo; 2007.

7. Osório FL, Crippa JAS, Loureiro SR. Instrumentos de avaliação do transtorno de ansiedade social. Rev Psiq Clín. 2005;32:73-83.

8. Osório FL, Crippa JAS, Loureiro SR. Instruments for the evaluation of social phobia. In: Axelby CP, editor. Social phobia: etiology, diagnoses and treatment. Hauppauge: Nova Science Publishers; 2008. p. 150-83.

9. Hofmann SG, DiBartolo PM. An instrument to assess self-statements during public speaking: scale development and preliminary psycometric properties. Behav Res Ther. 2000;31:499-515. 
10. Gerlach AL, Heinrichs NC, Zimmerman T. SSPS: a German version of the questionnaire to assess the cognitive components of fear of public speaking. Z Klin Psychol Psychother. 2007;36:112-20.

11. Gallego MJ, Botella C, Garcia-Palacios A, Quero S, Baños RM. Self-statements during Public Speaking Scale (SSPS): psychometric properties in a Spanish sample of social phobia. Behav Psychol. 2010;18:343-63.

12. Rivero R, Garcia-Lopez LJ, Hofmann SG. The Spanish version of the self-statements during public speaking scale: validation in adolescents. Eur J Psychol Assess. 2010;26:129-35.

13. Osório FL, Crippa JAS, Loureiro SR. Escala para autoavaliação ao falar em público (SSPS): validação transcultural para o português do Brasil, estudo dos itens e da consistência interna em amostra da população geral de universitários. Rev Psiq Clín. 2009a;35:207-11.

14. Connor KM, Kobak KA, Churchill LE, Katzelnick D, Davidson JR. Mini-Spin: a brief screening assessment for generalized social anxiety disorder. Depress Anxiety. 2001;14:137-40.

15. First MB, Spitzer RL, Gibon M, Willians JBW. Structured Clinical Interview for DSM-IV Axis I Disorders - Clinician Version (SCID-CV). Washington, DC: American Psychiatric Press; 1997.

16. Connor KM, Davidson JR, Churchill LE, Sherwood A, Foa E, Weisler RH. Psychometric properties of Social Phobia Inventory (SPIN). Br J Psychiatry. 2000;176:379-86.

17. Osório FL, Crippa JAS, Loureiro SR. Transcultural validation of the Brazilian Portuguese version of the Social Phobia Inventory (SPIN): study of the items and internal consistency. Rev Bras Psiquiatr. 2009b;31:25-9.

18. Osório FL, Crippa JAS, Loureiro SR. A study of the discriminative validity of a screening tool (MINI-SPIN). Eur Psychiatry. 2007;22:239-49.

19. Beck AT, Steer RA. Beck Anxiety Inventory: manual. San Antonio: Psychological Corporation; 1993.
20. Cunha JA. Manual da versão em português das Escalas Beck. São Paulo: Casa do Psicólogo; 2001.

21. Davidson JR, Potes NL, Richichi EA, Ford SM, Krisnan KR, Smith RD, Wilson W. The Brief Social Phobia Scale. J Clin Psychiatry. 1991;52:48-51.

22. Osório FL, Crippa JAS, Loureiro SR. Cross-cultural validation of the Brief Social Phobia Scale for use in Portuguese and the development of a structured interview guide. Rev Bras Psiquiatr. 2006;28:212-7.

23. Del-Ben CM, Vilela JAA, Crippa JAS, Hallak JEC, Labate CM, Zuardi AW. Test-retest reliability of the Structured Clinical Interview for DSM-IV - Clinical Version (SCID-CV) translated into Portuguese. Rev Bras Psiquiatr. 2001;23:156-9.

24. Crippa JAC, Osório FL, Del Ben CM, Santos-Filho A, Silva-Freitas MC, Loureiro SR. Comparability between telephone and face-to-face structured clinical interview for DSM-IV (SCID) in assessing social anxiety disorder. Perspect Psychiatr Care. 2008;44:241-7.

25. Streiner DL, Norman GR. Health measurement scales: a practical guide to their development and use. 3th ed. London: Oxford University Press; 2003.

26. Hair JF, Anderson RE, Tatham RL, Black WC. Análise multivariada de dados. 5. ed. Porto Alegre: Bookman; 2005.

27. Floyd FJ, Widaman KF. Factor analysis in the development and refinement of clinical assessment instruments. Psychol Assess. 1995;7:286-99.

28. Arrais KC, Sousa JP, Tresniak CF, Santos A, Freitas MCS, Osório FL, et al. Social anxiety disorder women easily recognize fearful, sad and happy faces: the influence of gender. J Psychiatr Res. 2010;44(8):535-40. Epub 2009 Dec 4.

29. Schneier FR, Johnson J, Homig CD, Liebowitz MR, Weissman MM. Social phobia: comorbidity and morbidity in an epidemiologic sample. Arch Gen Psychiatry. 1992;49:282-8. 